\title{
Características da reação tipo 1 e associação com vírus B e C da hepatite na hanseníase
}

\author{
Type 1 reaction in leprosy: characteristics and association \\ with hepatitis B and C viruses
}

\author{
Vitória P.A. Rego ${ }^{1}$, Paulo R.L. Machado ${ }^{2}$, Isabela Martins ${ }^{1}$, \\ Ronald Trindade ${ }^{3}$ e Raymundo Paraná ${ }^{3}$
}

\begin{abstract}
RESUMO
O episódio reacional tipo 1 ou reação reversa é ocorrência inflamatória aguda que atinge a pele e nervos periféricos, encontrada em até $30 \%$ dos pacientes com hanseníase, sendo causa comum de incapacidade física. Fatores de risco associados incluem uso de poliquimioterapia e infecções virais. Neste estudo, foram avaliados 620 pacientes com hanseníase. Reação reversa foi diagnosticada em 121 (19,5\%) casos, sendo mais freqüente nos indivíduos borderlines (48\%). Início da poliquimioterapia foi considerado fator de risco para reação reversa, com $52 \%$ dos casos apresentando o primeiro episódio neste momento. Neurite foi documentada em $73 \%$ dos casos. A presença de vírus B ou C da hepatite foi documentada em $9 \%$ de 55 pacientes com reação reversa e em nenhum dos 57 pacientes sem reação $(p=0$, 026; teste exato de Fisher), sugerindo possivel papel destes agentes como fatores de risco para desenvolvimento de reação reversa na hanseníase.
\end{abstract}

Palavras-chaves: Hanseníase. Reação reversa. Episódio reacional tipo 1. Vírus B. Vírus C.

\begin{abstract}
Type 1 reaction or reversal reaction is an acute inflammatory episode in the skin and peripheral nerves that is found in up to $30 \%$ of leprosy patients and commonly causes physical disabilities. Multidrug chemotherapy and viral infections are associated risk factors. In this study, 620 leprosy patients were evaluated. Reversal reactions were diagnosed in 121 cases (19.5\%) and were most frequently found in borderline patients (48\%). Starting on multidrug chemotherapy was considered to be a risk factor for reversal reaction: $52 \%$ of the cases presented their first episode at this time. Neuritis was found in $73 \%$ of the cases. The presence of hepatitis B or C virus was documented in 9\% of the 55 patients with reversal reaction, while it was not detected in any of the 57 patients without reaction $(p=0.026$, Fisher's exact test). This suggests that these agents may have a role as risk factors for developing reversal reactions.
\end{abstract}

Key-words: Leprosy. Reversal reaction. Type 1 reaction. Hepatitis B virus. Hepatitis C virus.

A hanseníase, doença crônica infecciosa causada pelo Mycobacterium leprae, pode originar o aparecimento de graves sequielas e deformidades, devido ao comprometimento do sistema nervoso periférico. A presença de processo inflamatório agudo atingindo pele e nervo periférico, conhecido como episódio reacional tipo 1 (ER1) ou reação reversa (RR), é o principal responsável pela neurite hansênica, causa direta de incapacidade física.

A reação reversa e a neurite aguda constituem emergência na hanseníase, são de alta morbidade e difícil manejo e requerem tratamento rápido devido ao risco de levar a dano neural permanente. A reação reversa ocorre mais freqüentemente nas formas borderline ${ }^{6}{ }^{16}$, atingindo 8 a $30 \%$ dos pacientes ${ }^{6} .0$ episódio reacional tipo 1 está associado à ativação de mecanismo de hipersensibilidade tardia contra antígenos do Mycobacterium lepra ${ }^{10}$, resultando em dano tecidual. Caracteriza-se pela exacerbação de lesões pré-existentes, surgimento de novas lesões, episódios de neurite graves, com espessamento neural doloroso à palpação ou dor espontânea, podendo se acompanhar de febre, mal estar e anorexia, além de edema especialmente nas mãos e pés ${ }^{1719}$.

Fatores de risco para o desenvolvimento da RR têm sido bem documentados tais como formas borderline ${ }^{67}$, infecções virais ${ }^{16}$, instituição de poliquimioterapia (PQT) ${ }^{16}$ extensão da doença ${ }^{14}$ e vacinação com $\mathrm{BCG}^{6}$.

\footnotetext{
1. Serviço de Dermatologia, Hospital Universitário Prof. Edgar Santos, Universidade Federal da Bahia, Salvador, BA. 2. Serviço de Imunologia, Hospital Universitário Prof. Edgar Santos, Universidade Federal da Bahia, Salvador, BA. 3.Serviço de Hepatologia, Hospital Universitário Prof. Edgar Santos, Universidade Federal da Bahia, Salvador, BA.

Endereço para correspondência: Dr. Paulo Roberto L. Machado. Serviço de Imunologia. Hospital Universitário Prof. Edgard Santos/UFBA. Rua João das Botas s/n, $5^{\circ}$ andar, Canela, 40110-160 Salvador, BA.

Fax: 55-71 3245-7110

e-mail: prlmachado@uol.com.br

Recebido para publicação em: 23/10/2004

Aceito em: 18/07/2007
} 
Embora a literatura demonstre co-infecção de pacientes portadores de hanseníase com vírus da hepatite B (VHB) e C $(\mathrm{VHC})^{3}$, a presença destes vírus como possíveis fatores associados ao surgimento de RR ainda não foi documentada.

0 conhecimento de fatores de risco associados à RR é fundamental para intervenção terapêutica precoce, diminuindo o aparecimento de seqüelas graves e irreversíveis na hanseníase. Neste estudo, a frequiência e as características clínicas da RR em pacientes ambulatoriais de hanseníase são descritas. Adicionalmente, a identificação de fatores de risco associados a manifestações clínicas ou à co-infecção pelo VHB ou VHC, que possam estar relacionados ao surgimento de RR, foram investigados.

\section{MATERIAL E MÉTODOS}

0 presente estudo foi realizado através da avaliação de 620 pacientes matriculados no Ambulatório de Hanseníase do Hospital Universitário Prof. Edgar Santos, durante o período de 1992 a 2003, retrospectivamente através do levantamento de prontuários até 2001, e a partir daí de maneira prospectiva até 2003. Os pacientes foram classificados de acordo com os critérios de Ridley e Jopling ${ }^{13}$ nas formas: tuberculóide (TT), borderline tuberculóide (BT), borderline borderline (BB), borderline lepromatosa (BL) e lepromatosa (LL). Pacientes com a forma inicial (indeterminada) também foram incluídos. Todos os pacientes com diagnóstico clínico de hanseníase confirmados por exame baciloscópico e/ou histopatológico foram estudados. 0 índice baciloscópico inicial foi determinado após coleta em quatro regiões diferentes, variando de 0 a 4,25.

0 diagnóstico de RR foi realizado segundo critérios clínicos como surgimento de novas lesões, aparecimento de eritema e tumefação nas lesões pré existentes, neurite e edema das mãos.e dos pés. Estes pacientes foram analisados quanto à idade, gênero, forma clínica, índice baciloscópico, presença de neurite e ou lesões de pele, relação da reação com PQT, e sintomas associados como febre e astenia.

Pacientes do ambulatório que não apresentavam RR no momento do atendimento e até o final do tratamento foram selecionados como grupo de comparação. Ficha com dados gerais do paciente e da doença foram preenchidas de todos os pacientes, assim como um questionário geral sobre dados epidemiológicos para vírus B e C da hepatite.

Em 55 pacientes que cursavam com RR e em 57 pacientes com hanseníase do grupo de comparação, sorologia pelo método de ELISA foi realizada para vírus B- AgHBS e Anti-HBC (Organon ${ }^{\circledR}$, Holanda) e vírus C- Anti-HCV (Organon ${ }^{\circledR}$, Holanda) da hepatite.

Os dados foram registrados e analisados pelo programa SPSS (versão 9.0), com utilização do teste exato de Fisher na análise das variáveis.

Todos os pacientes selecionados prospectivamente assinaram o Termo de Consentimento Informado Livre e Esclarecido aprovado pelo Comitê de Ética em Pesquisa do Hospital Universitário Prof. Edgar Santos.
A partir dos resultados das sorologias para VHB e VHC, todos os pacientes com resultados positivos foram encaminhados ao serviço de Hepatologia do mesmo Hospital para acompanhamento.

\section{RESULTADOS}

Dos 620 pacientes portadores de hanseníase acompanhados, 121 (19,5\%) foram diagnosticados com RR. A distribuição das formas clínicas nos pacientes com RR mostrou predomínio das formas borderline com 58(48\%) pacientes A Tabela 1 mostra as características demográficas e clínicas dos pacientes com RR. 0 aparecimento do ER1 foi mais comum no sexo feminino (55,4\%), ocorrendo preferencialmente nos indivíduos adultos com idade média de 43,3 $\pm 19,3$ anos.

Tabela 1 - Características demográficas e clínicas dos pacientes com reação reversa.

\begin{tabular}{|c|c|c|}
\hline Características & Número & Percentagem \\
\hline \multicolumn{3}{|l|}{ Gênero } \\
\hline feminino & 67 & 55,4 \\
\hline masculino & 54 & 44,6 \\
\hline Idade (anos) & $43,3 \pm 19,3$ & \\
\hline Índice bacilar & $0,63 \pm 1,1$ & \\
\hline \multicolumn{3}{|l|}{ Forma clínica } \\
\hline lepromatosa & 5 & 4,1 \\
\hline borderline & 58 & 48,0 \\
\hline tuberculóide & 38 & 31,4 \\
\hline indeterminada & 20 & 16,5 \\
\hline \multicolumn{3}{|l|}{ Relação com a PQT } \\
\hline antes & 34 & 28,1 \\
\hline durante & 63 & $52,1^{*}$ \\
\hline pós & 24 & $19,8^{*}$ \\
\hline \multicolumn{3}{|l|}{ Dados clínicos } \\
\hline neurite & 88 & 72,7 \\
\hline lesão de pele & 54 & 44,6 \\
\hline astenia & 7 & 5,8 \\
\hline febre & 2 & 1,6 \\
\hline
\end{tabular}

A poliquimioterapia mostrou-se fator de risco importante para RR, com apenas $28 \%$ dos casos ocorrendo antes do seu início. Em 87 (72\%) pacientes o primeiro episódio reacional se manifestou durante $(52 \%)$ ou após $(20 \%)$ o uso da PQT $(p=0,019)$.

A manifestação clínica mais comum nos pacientes com RR foi a presença de neurite uni ou bilateral em $73 \%$ dos casos. A presença de lesões cutâneas inflamatórias características foi determinada em $45 \%$. Outros sintomas clínicos associados como febre e astenia estavam presentes num pequeno número de pacientes (Tabela 1 ).

A análise do soro de 55 pacientes com ER1 e 57 pacientes do grupo controle com relação à presença de infecção por VHB ou VHC é mostrada na Tabela 2. Foi documentada positividade para VHB em 2 (3,6\%) pacientes com RR e para VHC em 3 (5,5\%) 
Tabela 2 - Resultado da sorologia para virus da bepatite B e virus da bepatite $C$ nos pacientes com reação reversa.

\begin{tabular}{|c|c|c|c|c|c|c|}
\hline \multirow[b]{2}{*}{ Situação } & \multicolumn{2}{|c|}{ VHB } & \multicolumn{2}{|c|}{$\mathrm{VHC}$} & \multicolumn{2}{|c|}{$\mathrm{VHB}+\mathrm{VHC}$} \\
\hline & $\mathrm{n}^{\underline{0}}$ & $\%$ & $\mathrm{n}^{0}$ & $\%$ & $\mathrm{n}^{\underline{0}}$ & $\%$ \\
\hline Pacientes com RR & $2 / 55$ & 3,6 & $3 / 55$ & 5,5 & $5 / 55$ & $9,1^{*}$ \\
\hline Pacientes sem RR & \multicolumn{2}{|c|}{$0 / 57$} & \multicolumn{2}{|c|}{$0 / 57$} & \multicolumn{2}{|c|}{$0 / 57^{*}$} \\
\hline
\end{tabular}

* A análise levou em consideração que os indivíduos não apresentavam co-infecção VHB e VHC; $p=0,026$, teste exato de Fisher.

VHB: vírus da hepatite $\mathrm{B}$, VHC: vírus da hepatite $\mathrm{C}$

RR: reação reversa

indivíduos. Nenhum paciente no grupo controle apresentou teste positivo para VHB ou VHC.

A co-infecção com VHB ou VHC torna-se fator de risco significante $(\mathrm{p}=0,026)$ com odds ratio de $10 \%$ quando comparamos o número total de sorologias positivas para VHB ou VHC no grupo RR $(9,1 \%)$ com o grupo controle.

\section{DISCUSSÃO}

Este trabalho analisa a presença e as características da RR numa população de 620 pacientes ambulatoriais de hanseníase na Cidade de Salvador, Bahia. A ocorrência de RR em 19,5\% dos nossos pacientes, assim como a predominância em pacientes borderline ( $48 \%$ dos casos) demonstra a alta frequiência deste evento e sua predileção por determinadas formas clínicas. Estes dados encontram respaldo na literatura que descreve a ocorrência de ER1 em 8 a 30\% dos pacientes com hanseníase, sendo mais comum nos pacientes do grupo borderline ${ }^{6716}$. Avaliação retrospectiva de 388 pacientes no Nepal demonstra que pacientes borderlines e pacientes com formas extensas da doença são os que apresentam maior chance de desenvolverem $\mathrm{RR}^{20}$.

Saunderson, em $2000^{16}$ demonstrou que pacientes jovens do sexo feminino apresentam maior risco para episódios tardios de RR, enquanto Scollard e cols ${ }^{18}$ na avaliação de 178 pacientes apontam o sexo feminino como risco significante para RR. A análise do gênero e faixa etária em nosso estudo mostra 54 (44,6\%) pacientes do sexo masculino, e 67 (55,4\%) do sexo feminino, que mesmo em predomínio não se mostrou variável preditora para o desenvolvimento de RR. A média de idade dos nossos pacientes com RR foi de 43 anos, quando os indivíduos estão na faixa etária mais produtiva, o que ressalta a importância da implementação de medidas que facilitem o diagnóstico precoce e a prevenção de incapacidades.

O início do tratamento da hanseníase com PQT tem sido implicado no desencadeamento de ER1, o que parece acontecer devido à liberação de antígenos pela morte dos bacilos ${ }^{20}$. 0 início e o primeiro ano de terapia são fatores de risco identificados em mais de um estudo ${ }^{1617}$. Os nossos resultados ratificam os da literatura, com 63 (52\%) dos pacientes apresentando o primeiro episodio de RR durante o tratamento com PQT.

Considerando que o potencial incapacitante do ER1 se deve ao comprometimento dos nervos periféricos, nosso dado mostrando que $73 \%$ destes pacientes apresentavam neurite uni ou bilateral ressalta a importância de considerar a RR como a principal causa de lesão do nervo na hanseníase.

A identificação de pacientes com maior possibilidade de desencadear RR pode ter implicações importantes na redução da morbidade e do aparecimento de seqüelas, consequiência comum do episódio reacional ${ }^{12}$. Assim, o estudo de fatores de risco que se associem com o aparecimento de RR pode ser fundamental para evitar o desencadeamento destes episódios ou facilitar o diagnóstico e intervenção precoces. Diversas outras características clinicas e epidemiológicas tem sido associadas como fatores de risco para RR, a exemplo de estresse intenso, vacinação, infecções virais e gravidez.

Trabalhos visando identificar vírus como HIV e HTLV-I e possível correlação com a hanseníase tem sido motivo de publicações em vários países onde a hanseníase é endêmica, sem contudo demonstrar associação evidente ${ }^{811}$. No entanto, co-infecção com HIV foi associada a alto risco para aparecimento de eritema nodoso hansênico e possivelmente a um aumento na recorrência de $\mathrm{RR}^{4}$.

A possível associação entre hanseníase e vírus $\mathrm{B}$ e $\mathrm{C}$ da hepatite tem sido estudada por diversos autores, principalmente na África e no Japão. A maior parte dos estudos encontra associação entre hanseníase e o VHC, variando de $7,15 \%$ a $18 \%$ principalmente em pacientes institucionalizados ${ }^{23}$. No Brasil, em estudo realizado no Estado de Goiás a prevalência de VHC em 216 pacientes foi de $1,8 \%{ }^{15}$. No entanto, o papel destes agentes como fator de risco para o desencadeamento ou agravamento das reações não tem sido relatado. Neste trabalho, sorologia positiva para VHB ou VHC foi documentada em 9,1\% dos pacientes com RR em contraste com ausência de evidência de infecção no grupo controle de pacientes com hanseníase sem RR. Este dado merece atenção, tendo em vista a possibilidade da interação entre estes agentes e a resposta imunológica dos pacientes hansênicos predispondo ao aparecimento de RR.

No Brasil, onde a hanseníase ainda é sério problema de saúde pública em regiões também endêmicas para o VHB e/ou VHC, a exemplo da Bahia, a verificação de nossos dados em número maior de pacientes torna-se fundamental.

\section{REFERÊNCIAS}

1. Becx-Bleumink M, Berhe D. Occurence of reactions, theis diagnosis and management leprosy patients treated with multidrug therapy; experience in leprosy control program of the All Africa Leprosy and Rehabilitation Training Center (ALERT) in Ethiopia. International Journal of Leprosy and Other Mycobacterial Diseases 60: 173-84, 1992.

2. Denis F, Aussel L, Ranger S, Martin P, Itoua-N Gaporo A,Frommel D, TeckleHaimanot RT, Sangare A, MBoup S, Milian J Prevalence of antibodies to hepatitis $\mathrm{C}$ virus among patients with leprosy in several Africa Countries an the Yemen. Journal of Medical Virology 43: 1-4, 1994

3. Egawa K, Yukawa T, Arakawa S, Tanaka T, Tsuda F, Okamoto H, Miyakawa Y, Mayumi M. Hepatitis C virus antibody, viral RNA and genotypes in leprous patients in Japan. Journal of Hepatology 196: 397-402, 1995.

4. Gebre S, Saunderson P, Messele T, Byass P. The effect of HIV status on the clinical picture of leprosy: a prospective study in Ethiopia. Leprosy Review 71: 338-343, 2000. 
5. Joseph MS Unilateral edema of reversal reaction (RR) in borderline leprosy. Leprosy Review 65: 9-33, 1994.

6. Lienhardt C, Fine PE. Type 1 reaction, neuritis and disability in leprosy. What is the current epidemiological situation? Leprosy Review 65: 9-33, 1994.

7. Lockwood DN, Vinayakumar S, Stanley JN, Mc-Adam KP, Colston MJ. Clinical features and outcome of reversal (type 1) reactions in Hyderabad, India. International Journal of Leprosy and Other Mycobacterial Diseases 61: 8-15, 1993.

8. Machado P, David Y, Pedroso C, Brittes C, Barral A, Barral-Netto M.Leprosy and HIV Infection in Bahia, Brazil. International Journal of Leprosy and Other Mycobacterial Diseases 66: 227-229,1998.

9. Martelli Celina.Associação entre Hanseníase e infecção pelo vírus da hepatite B:estudo de caso controle. Tese de Doutorado, Faculdade de Saúde Publica, Universidade de São Paulo, São Paulo, 1995

10. Modlin RL. In situ characterization of T lymphocyte subsets in the reactional states of leprosy. Clinical Experimental. Immunology 53: 17-24, 1983.

11. Muneish H, Taguchi H, Sawada T, Ikezoe T, Matsui S, Tanaka S, TaniguchiT, Onoue 0 , Miyoshi I. Prevalence of HTLV-I in leprosy patients in two sanatoriums in Japan. Journal Acquired Immune. Deficience Syndrome Human Retrovirology 17: 380-383, 1998.

12. Nery J, Garcia C, Wanzeller S, Sales A, Gallo M, Vieira L. Características clínicohistopatológicas dos estados reacionais na Hanseníase em pacientes submetidos à poliquimioterapia (PQT). Anais Brasileiros de Dermatologia 74: 27-33, 1999.
13. Ridley DS, Jopling WH. Classification of leprosy according to immunity. A five group system. International Journal of Leprosy and Other Mycobacterial Diseases 34: 255-273, 1996.

14. Roche PW, Le Master J, Butlin R. Risk factors for type 1 reactions in leprosy. International Journal of Leprosy and Other Mycobacterial Diseases 65: 450-455, 1997.

15. Rosa H, Martins R, Vanderborght B. Short report: association between leprosy and hepatitis $\mathrm{C}$ infections: a survey in a region Central Brazil. The American Journal of Tropical Medicine and Hygiene 55: 22-23, 1996.

16. Saunderson P. The epidemiology of reactions and nerve damage. Leprosy Review 71: $\$ 106-\$ 110,2000$.

17. Schreuder PAM. The occurrence of reations and impairments in leprosy: experience in the leprosy control program of three provinces in northeastern Thailand, 1978-1995. II. Reactions. International Journal of Leprosy and Other Mycobacterial Diseases 66: 159-169, 1998.

18. Scollard DM, Smith T, Bhoopat L, Theetranont C, Rangdaeng S, Morens DM. Epidemiologic characteristics of leprosy reactions. International Journal of Leprosy and Other Mycobacterial Diseases 62: 559-567, 1994.

19. Valentini A, Nery JAC, Salles AM. Edema na hanseníase: aspectos clínicos e terapêuticos. Revista da Sociedade Brasileira de Medicina Tropical 32: 131-138, 1999.

20. Van Brakel WH, Khawas IB. Lucas SB. Reactions in leprosy: an epidemiological study of 386 patients in the west Nepal. Leprosy Review 6: 190-203, 1994. 